\title{
Predictable looping of the developing vertebrate gut explained
}

A

$\mathrm{n}$ interdisciplinary team of researchers from Harvard has shed light on why the developing vertebrate gut tube loops in a predictable way that is conserved within a given species, giving credence to the mathematical and physical approach to biological shape that was championed by D'Arcy Thompson in his 1917 book On Growth and Form. Using developmental experiments, a physical mimic, a mathematical theory and a computational model, they have shown that it is differences in the growth rate of the gut tube and the dorsal mesentery that forces the gut tube to coil.

"When Cliff Tabin and Natasza Kurpios showed me images of the gut, I hypothesized that differential growth between the gut and mesentery was responsible for the pattern and was able to construct a theory for the size and shape of the loops," explains L. Mahadevan, corresponding author. "One might think that the gut coils because it is restricted by the size of the abdominal cavity. That's not actually the case," says coauthor Thierry Savin. "The gut coils because when the mesentery and the gut tube grow at different rates while remaining attached, coiling is the only possible result."

Developmental experiments excluded the possibility that external spatial packing constraints were responsible for the predictable looping. When the gut tube and dorsal mesentery were surgically dissected from chick embryos, not only did the loops remain in place but their structure was also identical to that seen in ovo at multiple developmental stages. The idea that asymmetrical proliferation of cells present in the bends of the gut tube could be responsible for the looping was also ruled out, as the number of mitotic cells present was consistent across the gut independent of developmental stage.

The role of the dorsal mesentery, which attaches the gut tube to the body,
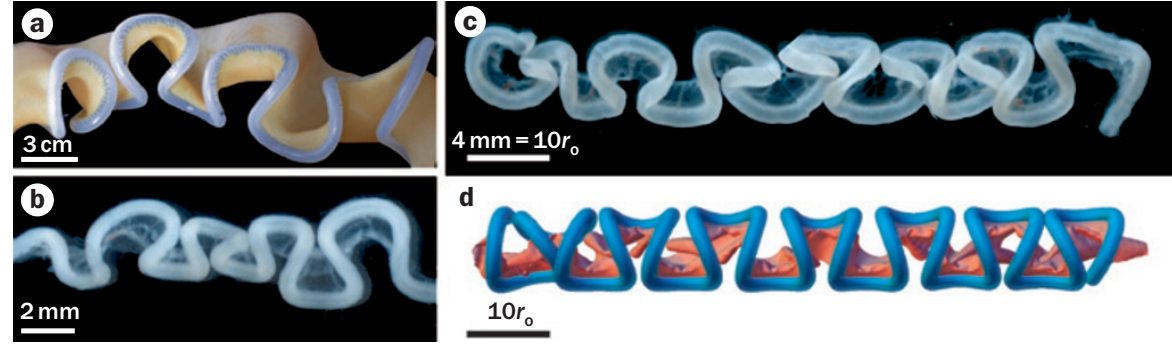

The structure of the relaxed physical model (a) is very similar to the structure of the chick gut (b) shown at embryonic day 12 (E12). The chick gut at E16 (c) and its simulated counterpart (d). () Savin, T. et al. Nature 476, 57-62 (2011).

was investigated by separating the chick dorsal mesentery from the gut tube at embryonic day 14 (E14). After separation, the gut tube uncoiled into a straight tube, whereas the dorsal mesentery contracted, suggesting the gut tube was being compressed and the dorsal mesentery was under tension. Surgical separation of part of the dorsal mesentery from the gut in ovo at E4, prior to gut loop development, revealed an absence of gut loops in only those regions of the E12 chick gut in which the mesentery and gut had been separated.

As the dorsal mesentery was then shown to grow uniformly, as does the gut tube, it confirmed to the team "...that the uniform differential growth between the gut and the mesentery could be at the origin of loop formation. Because the gut tube is slender, with a length that is much larger than its radius, it responds physically to the differential strain-induced compression from the attached mesentery by bending and looping..."

This naturally suggested a physical model of the looping in terms of a latex sheet (mesentery) and a silicone tube (gut tube). The latex sheet was stretched along the length of unstretched silicone tube and the two stitched together. Using the model, it was possible to simulate the differential strain placed on the gut tube and dorsal mesentery by their differential growth. When the composite was allowed to relax, the silicone tube looped in a very similar way to the in ovo chick gut.
Bolstered by this and armed with mathematical scaling laws for the loop period, radius and number, the team generated predictions for gut looping at different stages of chick development in terms of the measurable geometry, elasticity and strain mismatch. The data also allowed for detailed numerical simulation of gut looping. "Our model captures the salient properties of the looping patterns ... strongly suggesting that the main features of the chick gut looping are established by the simple balance of forces induced by the relative growth between the gut and the mesentery," say the authors.

Finally, the researchers tested their computational model in other speciesquail, zebra finch and mice. They confirmed the accuracy of the predictions with geometrical and biophysical data collected from these species. "A simple mathematical scaling theory combined with computations showed that these patterns are quantitatively predictable using experimentally derived measurements with no adjustable parameters. This allows for a study of the pattern as a function of developmental time-and even across species," concludes Mahadevan.

Natalie J. Wood

Original article Savin, T. et al. On the growth and form of the gut. Nature 476, 57-62 (2011) 